\title{
The imprinted IncRNA Peg13 regulates sexual preference and the sex-specific brain transcriptome in mice
}

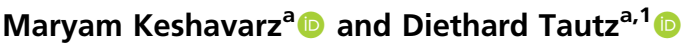 \\ aDepartment of Evolutionary Genetics, Max Planck Institute for Evolutionary Biology, 24306 Plön, Germany
}

Edited by Jeannie T. Lee, Massachusetts General Hospital, Boston, MA, and approved January 25, 2021 (received for review November 3, 2020)

\begin{abstract}
Mammalian genomes include many maternally and paternally imprinted genes. Most of these are also expressed in the brain, and several have been implicated in regulating specific behavioral traits. Here, we have used a knockout approach to study the function of Peg13, a gene that codes for a fast-evolving IncRNA (long noncoding RNA) and is part of a complex of imprinted genes on chromosome 15 in mice and chromosome 8 in humans. Mice lacking the $3^{\prime}$ half of the transcript look morphologically wild-type but show distinct behavioral differences. They lose interest in the opposite sex, instead displaying a preference for wild-type animals of the same sex. Further, they show a higher level of anxiety, lowered activity and curiosity, and a deficiency in pup retrieval behavior. Brain RNA expression analysis reveals that genes involved in the serotonergic system, formation of glutamatergic synapses, olfactory processing, and estrogen signaling-as well as more than half of the other known imprinted genes-show significant expression changes in Peg13-deficient mice. Intriguingly, these pathways are differentially affected in the sexes, resulting in male and female brains of Peg13-deficient mice differing more from each other than those of wild-type mice. We conclude that Peg13 is part of a developmental pathway that regulates the neurobiology of social and sexual interactions.
\end{abstract}

imprinted genes | behavior | partner preference | brain transcriptome

$\mathbf{M}$ ammalian genomes include around a hundred genes that are fully or preferentially expressed from the maternal or paternal chromosomes. Many of them occur in clusters which are epigenetically marked in the gametes to generate the asymmetric expression after fertilization (1). Initial studies on such imprinted genes have mostly focused on their effects during embryogenesis, but most are also expressed in the brain. Dedicated knockout studies have shown that these brain-expressed genes are involved in regulating specific behavioral traits, including maternal care $(2,3)$, social dominance $(4,5)$, reactivity to novel environments $(6,7)$, or foraging $(8)$. In a previous study on assortative mating preferences between wild-type (WT) populations of Mus musculus domesticus mice, we found a paternal influence on mate choice patterns in seminatural environments (9). In a subsequent analysis, we identified two imprinted loci as possible candidates to influence this mate choice pattern; they are expressed from the paternal chromosome and evolve particularly fast between mouse populations (10). One of these loci is Peg13, a single-exon noncoding RNA gene on mouse chromosome 15 . It was originally identified as a locus with differential methylation of the paternal and maternal chromosomes (11).

Peg13 is expressed in many organs from the paternal allele only but with the highest expression throughout the brain, including olfactory bulbs and the vomeronasal organ $(10,12)$. It is located within an intron of $\operatorname{Trappc} 9$, a gene that shows a maternal expression bias (i.e., about $70 \%$ of the transcripts come from the maternal allele). Trappc 9 is flanked by three other imprinted coding genes: Kcnk9 with a strictly maternal expression, along with Chrac1 and Ago2 with maternally biased expression $(10,13)$
(Fig. 1). These four coding genes have clear orthologs in humans, but only Kcnk 9 shows maternal expression bias in humans, while the other three genes are equally expressed from both chromosomes (13). However, the differentially methylated region and $\mathrm{CpG}$ island close to the promotor region of the Peg13 transcript are conserved between humans and mice (14-16).

Here, we used a knockout approach to study the function of Peg13. Two knockout constructs were analyzed, one with a full deletion of the gene, the other with a deletion of the $3^{\prime}$ half. The full deletion is semilethal, while the partial deletion is fully viable and could therefore be analyzed in detail. We found that Peg13 has a major influence on behavioral patterns, especially with respect to sexual preference display. We further show that it is also involved in regulating the sex-specific expression of key regulatory genes in brain hormonal and synapse functions, as well as many other imprinted loci including some that are involved in regulating behavioral patterns. These findings establish Peg13 as being involved in a network required to set up the brain functions that regulate social interactions.

\section{Results}

Peg13 was identified as a candidate for the fast evolution of mating preferences between populations of house mice (10). To assess the overall rate of sequence divergence at the Peg13 locus, we generated an alignment with Peg13 sequences from closely related mouse populations, subspecies, and species (Fig. 1). We found that the substitution rates in the Peg13-transcribed region do not differ much from those of the flanking upstream and downstream noncoding regions, and that there is no indication of any more conserved blocks within the transcribed region. Given that Peg13 is a noncoding RNA, one cannot calculate $\mathrm{dN} / \mathrm{dS}$

\section{Significance}

Mammalian behavior is known to be influenced by genes that are expressed from only one of the parental chromosomes. The present study shows that mice carrying a specific deletion variant of the paternally expressed gene Peg13 prefer to associate with their own sex, along with additional behavioral changes. RNA expression analysis on the brains of these mice suggests that Peg13 is part of a regulatory network that governs the female-male differentiation of the brain, as well as the neurobiology of social interactions

Author contributions: M.K. and D.T. designed research; M.K. performed research; M.K. and D.T. analyzed data; and M.K. and D.T. wrote the paper.

The authors declare no competing interest.

This article is a PNAS Direct Submission.

This open access article is distributed under Creative Commons Attribution-NonCommercialNoDerivatives License 4.0 (CC BY-NC-ND).

${ }^{1}$ To whom correspondence may be addressed. Email: tautz@evolbio.mpg.de.

This article contains supporting information online at https://www.pnas.org/lookup/suppl/ doi:10.1073/pnas.2022172118/-/DCSupplemental.

Published March 3, 2021 

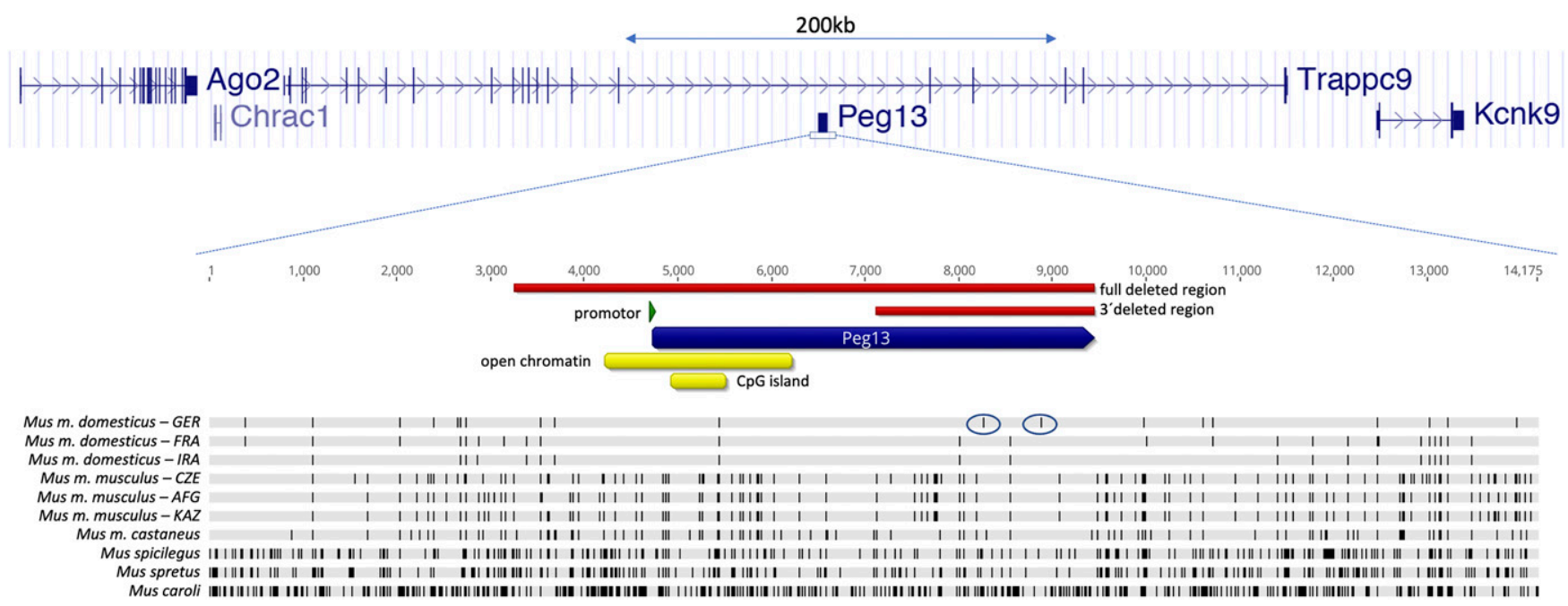

Fig. 1. Schematic view of the imprinted genes around the Peg13 gene in mice. The imprinted complex (region chr15:72,478,000 to $73,203,000$ in mm10) includes five transcripts: Ago2, Chrac1, Trappc9 (note that there are multiple annotated alternatively spliced transcripts for Trappc9, of which only the major one is shown), Peg13, and Kcnk9. The blow-up of the Peg13 region shows the region of open chromatin in the embryonic brain, as well as the CpG island (yellow blocks; annotations are derived from UCSC Browser tracks). The blocks that are deleted in the two knockout constructs are shown as red bars. (Bottom) An alignment against the mm10 mouse reference sequence with various mouse subspecies and species. Dashes indicate substitutions. The two differential substitutions between two mouse populations that led to our original identification of this candidate gene for differential mate choice (10) are located in the $3^{\prime}$ half of the transcript (circled with small ovals).

ratios, but the ratio of substitutions in the transcribed region to those of an equally sized downstream region is 0.7 (the average for all pairwise comparisons with the species shown in Fig. 1), suggesting an only slightly higher evolutionary constraint in the transcribed region. Therefore, Peg13 is already fast-evolving within mice, which can explain the lack of sequence conservation with humans.

Knockout Constructs. Taking the structural considerations concerning the $\mathrm{CpG}$ island within the transcribed region of Peg13 into account (Fig. 1), we constructed two versions of knockout alleles. The first removes only the $3^{\prime}$ part of the RNA, without affecting the $\mathrm{CpG}$ island (Peg13 ${ }^{\text {tm1Mev }}$ ). The second can be derived from the former allele through crossing to a strain that expresses Cre recombinase (17) and represents a complete deletion of the RNA (Peg13 $\left.3^{\text {tm 1.1Mevb }}\right)$, the CpG island, and part of the upstream region (Fig. 1 and SI Appendix, S1). However, we found that this full deletion is semilethal when inherited through the paternal side, while animals derived from crosses that harbored the full deletion on the maternal chromosome show no phenotype (see description in SI Appendix, S2).

In total, we obtained three animals harboring the deletion on the paternal chromosome. These showed a severe skin lesion phenotype, which did not allow us to do behavioral analysis, but we were still able to do RNA expression analysis in the brain. This indicated that deletion of the $\mathrm{CpG}$ island leads to a deregulation of the whole cluster, and the phenotype could be due to the misexpression of any of the genes in the cluster (see description in SI Appendix, S2). Hence, all the further analysis is based on the $3^{\prime}$-deletion allele only $\left(\right.$ Peg $\left.13^{\text {tm1Mevb }}\right)$. In the subsequent sections, we will designate this allele as $P e g 13^{-/-}$when both chromosomes are mutant, Peg $13^{+/-}$when the paternally transmitted chromosome is mutant, and $P e g 13^{-/+}$when the maternally transmitted chromosome is mutant.

$P e g 13^{-l-}$ animals were fully viable and showed no visible morphological phenotype. However, while monitoring their breeding performance, we noticed that breeding was impaired when the paternal allele was deficient (Table 1).
Analysis of breeding records during the establishment of the strain showed significantly fewer successful litters per breeding pair when both parents or the males were homozygous mutant Additionally, more litters were lost for combinations where only the female was homozygous mutant, or where the female and the male were mutant on the paternal chromosome (Table 1). While average litter sizes were not significantly different between the genotypes, there was a noticeable secondary loss of litters in pairs that were mutant for the paternal chromosome (Table 1).

Behavioral Tests. The relatively larger loss of litters among mutant mice prompted us to test whether maternal care is affected. For this, we used a standardized pup retrieval test (Materials and Methods) on eight mutant and WT animals each, all bred with WT males (i.e., the pups are expected to be WT, since they received the active allele from the father). Pup retrieval was defined as picking up a pup and transporting it to the nest. While all WT females retrieved the pups within minutes, most mutant females did not retrieve them at all during the 15-min observation period (Table 2). This indicates a deficiency in maternal care behavior, which could explain the high rate of secondary loss of litters.

To assess whether other behaviors are also impaired in $P e g 13^{-/-}$mice, we conducted a number of tests for anxiety, curiosity, and activity. We found significant differences between WT and mutant animals for each test (Table 2). The results suggest that $P e g 13^{-/-}$mice have higher anxiety, lower curiosity, and lower locomotor activity.

To confirm that these behavioral responses can be ascribed to the paternally transmitted chromosome, we repeated the behavioral tests for individuals that inherited the mutant allele from either the mother or the father. We found that significant differences could only be observed for animals that received the mutant allele from the father (Table 2), that is, the maternal allele does not contribute to the phenotypes.

Partner Preference. We used a previously developed partner preference test (18) as a proxy for mate choice behavior. This test is based on a four-way choice setup, where a central cage is 
Table 1. Breeding performance of Peg13 mutant mice

\begin{tabular}{|c|c|c|c|c|c|c|c|}
\hline Dam genotype* & $\begin{array}{c}\text { Sire } \\
\text { genotype* }\end{array}$ & $\begin{array}{l}\text { Total breeding } \\
\text { pairs, } n\end{array}$ & $\begin{array}{l}\text { Total pairs with } \\
\text { litters, } n\end{array}$ & $\begin{array}{l}\text { Surviving } \\
\text { litters, } n\end{array}$ & $\begin{array}{c}\text { Breeding success } \\
\text { rate }^{\dagger}\end{array}$ & $\begin{array}{c}\text { Average litter } \\
\text { size, } n\end{array}$ & $P$ value $^{\ddagger}$ \\
\hline$+/+(\mathrm{WT})$ & $+/+(\mathrm{WT})$ & 21 & 19 & 17 & 0.81 & 6 & \\
\hline$-/+$ & $-/+$ & 27 & 24 & 23 & 0.85 & 6 & 0.54 \\
\hline$+1-$ & $+1-$ & 11 & 4 & 2 & 0.18 & 7 & 0.056 \\
\hline$+/+$ & $-1-$ & 10 & 2 & 1 & 0.10 & 7 & 0.030 \\
\hline$-1-$ & $-1-$ & 33 & 12 & 4 & 0.12 & 6 & 0.0010 \\
\hline
\end{tabular}

*Genotypes are represented as maternal allele/paternal allele; the origin of the allele was determined by comparison of the genotypes of the parents in the breeding records during the establishment of the strain.

${ }^{\dagger}$ Ratio of live litters to total pairs, with only the first litter per pair included.

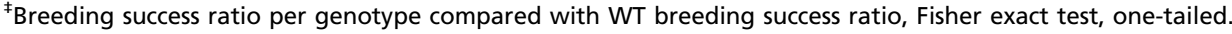

connected to four satellite cages via tubes with ring antennae for recording the movement of mice carrying an RFID (radio-frequency identification) tag (Fig. $2 A$ ). For each round of experiments, the focal mice were placed into the central cage and exposed for $3 \mathrm{~h}$ to the four satellite cages with mice of both sexes and different genotypes (wild type and knockout). Movement between the cages was tracked via the RFID signals, making it possible to determine the residence time in each of the cages.

Fig. $2 A$ shows the proportional time durations (in \%) that WT and Peg $13^{-1-}$ mutant mice spent in each of the cages in the first three 30-min intervals. WT mice (blue) showed the expected strong preference for the opposite sex, but more to the WT partner than to the mutant ones. Mutant mice (red), on the other hand, showed a strong preference for the same sex, especially mutant males for WT males. The effect was less pronounced for females and they associated similarly with WT and mutant partners. Further, the mutant mice were slower in making their choice. This latter effect can be quantified by plotting the general selectivity over time. This measure represents the strength of preference that focal mice show for satellite mice, independent of the direction of preference (Materials and Methods). The selectivity plots in Fig. $2 B$ show that WT animals displayed the highest selectivity in the first interval, while mutant animals displayed it only in the second interval. Males generally displayed higher selectivity than females. These observations suggest that the much lower breeding success of the mutant animals could be caused by changed sexual preference.

Transcriptome Analysis. A survey of the brains from WT and $P e g 13^{--}$mice in computed tomography (CT) scans showed no obvious structural differences (SI Appendix, S3). Therefore, we used a transcriptomic analysis to trace the effect of the Peg13 mutation on the regulatory networks in the brain. We extracted RNA from the whole brain of 16-wk-old animals, using eight individuals of each sex and each genotype (WT versus $P$ eg $13^{-1-}$ ). DESeq2 (19) was used to identify differentially expressed genes.

The loss of Peg13 gene function has a striking effect on the brain transcriptome of males and females. First, Peg $13^{-/-}$animals have overall a very different transcriptomic profile compared with WT mice (Fig. 3). Second, while WT brains do not differ between the sexes, the loss of Peg13 results in distinct male and female brain transcriptomes (Fig. 3).

About $15 \%$ of all tested transcripts $(8,004$ in males and 8,087 in females) showed significant differential expression between $P e g 13^{-/-}$and WT mice, with 5,899 overlapping between the sexes (Dataset S1 $A-C$ ). Within the imprinted cluster, Peg13 itself is still expressed, but about four- to eightfold less, as to be expected due to its shortened transcript and therefore also likely lowered transcript stability (note that the measured expression score is derived from the nondeleted $5^{\prime}$ part of the transcript, i.e., only about half of the reads are counted compared with the WT).

The Trappc9 gene, within which Peg13 is located, shows no significant expression difference. This suggests that the overall regulation of the imprinted cluster is not much affected by the $3^{\prime}$ deletion construct. Note that the full deletion has a profound effect on the expression of the flanking genes, suggesting that it does interfere with the regulation of the whole cluster, presumably due to the loss of the control region around the $\mathrm{CpG}$ island (SI Appendix, S2).

Table 2. Behavioral test score comparisons between Peg13 mutant and wild-type mice

\begin{tabular}{|c|c|c|c|c|c|}
\hline \multirow[b]{2}{*}{ Behavioral test } & \multirow[b]{2}{*}{ Score (test question) } & \multicolumn{4}{|c|}{ Genotype* } \\
\hline & & WT & $-1-$ & $-/+$ & $+/-$ \\
\hline Pup retrieval ${ }^{\dagger}$ & Time to retrieving displaced pups, min (maternal care) & $\begin{array}{c}n=160^{\star}, 169 \\
1.65 \pm 0.9\end{array}$ & $\begin{array}{c}\mathrm{n}=10 \AA, 109 \\
13.3 \pm 4 \\
\star * *\end{array}$ & $\begin{array}{c}n=60,59 \\
1.48 \pm 0.5 \\
\text { n.s. }\end{array}$ & $\begin{array}{c}n=60^{*}, 69 \\
12.4 \pm 5 \\
* * *\end{array}$ \\
\hline Open field ${ }^{\ddagger}$ & Average wall time, \% (anxiety) & $54 \pm 10$ & $\begin{array}{c}84 \pm 13 \\
* * *\end{array}$ & $\begin{array}{c}51 \pm 6 \\
\text { n.s. }\end{array}$ & $\begin{array}{c}78 \pm 15 \\
* \star *\end{array}$ \\
\hline Elevated plus maze ${ }^{\ddagger}$ & Average dark arm time, \% (anxiety) & $47 \pm 15$ & $\begin{array}{c}68 \pm 21 \\
* * *\end{array}$ & $\begin{array}{c}42 \pm 14 \\
\text { n.s. }\end{array}$ & $\begin{array}{c}69 \pm 11 \\
* * *\end{array}$ \\
\hline Novel object ${ }^{\ddagger}$ & Average time of the novel object, s (curiosity) & $71 \pm 18$ & $\begin{array}{c}28 \pm 12 \\
* * *\end{array}$ & $\begin{array}{c}69 \pm 16 \\
\text { n.s. }\end{array}$ & $\begin{array}{c}26 \pm 11 \\
* * *\end{array}$ \\
\hline
\end{tabular}

*Statistical tests (all compared with WT): ***P<0.001; n.s., nonsignificant; $t$ test, Bonferroni-corrected.

${ }^{\dagger}$ For females only, $n=8$ each for WT and $-/-; n=6$ each for $-/+$ and $+/-$ (note that the pups received the paternal allele from a WT male, i.e., are expected to have WT expression of Peg13).

${ }^{ \pm}$We found no sex-specific effects in these tests. 

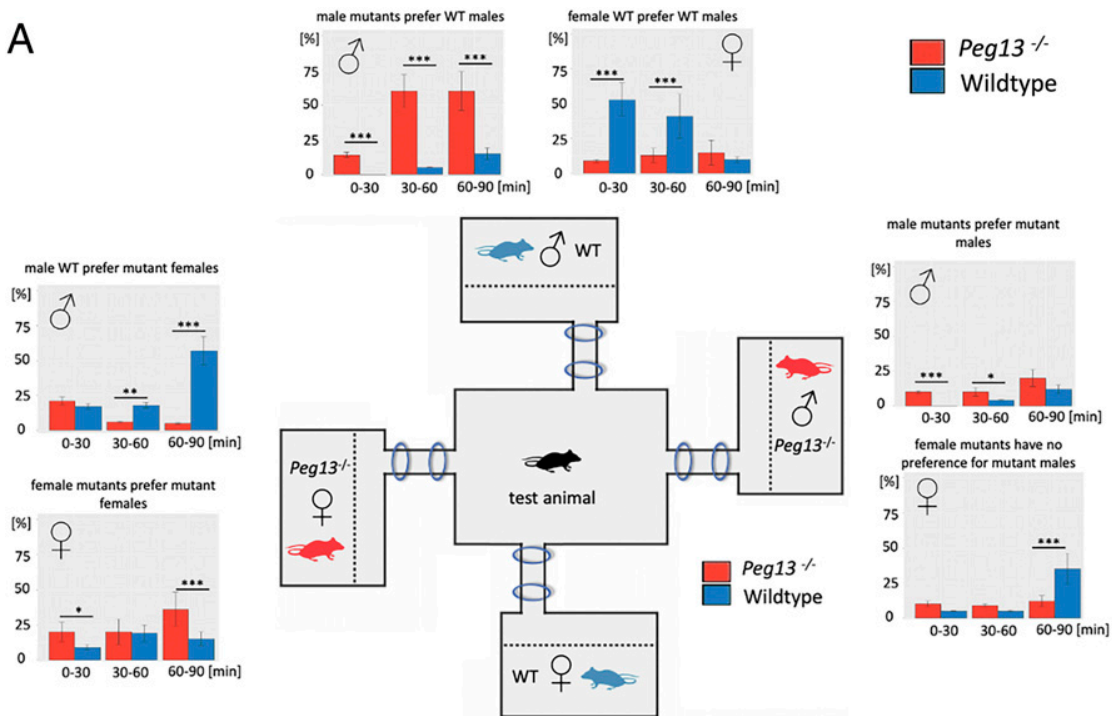

B
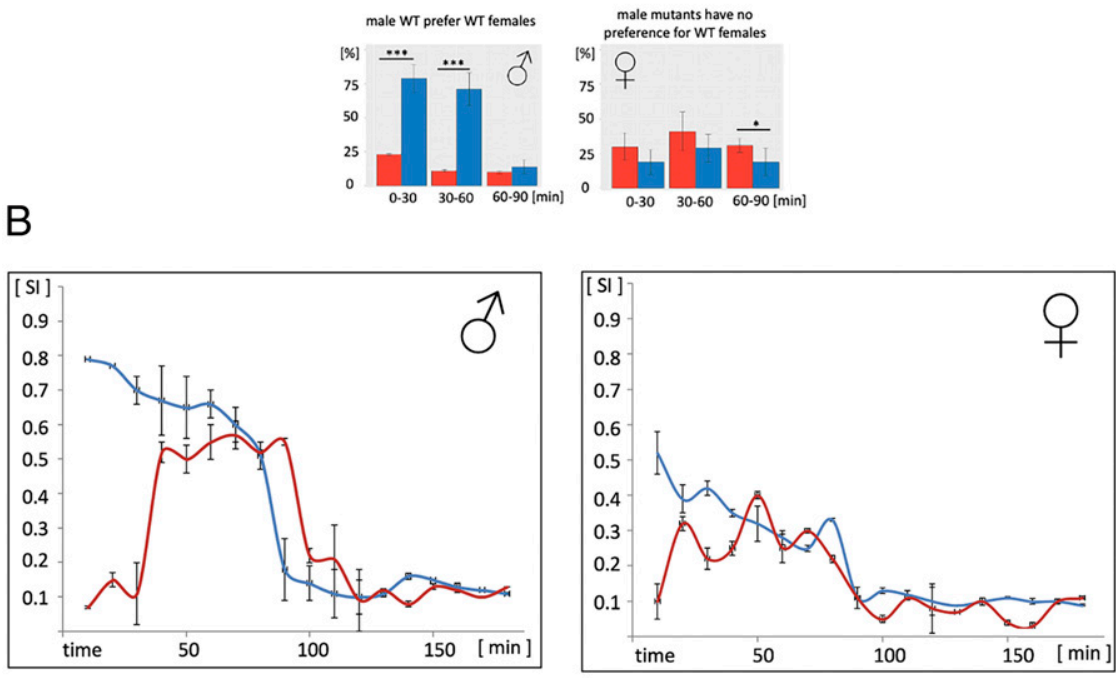

Fig. 2. Sex preference patterns of $P$ eg $13^{-/-}$(red) and wild-type (blue) mice. (A) Four-way choice test. The cage setup is shown (Center); the proportional times the focal mouse spent in the respective satellite and center cages are shown as bar plots for the first three 30-min time intervals. Error bars represent the variability of the proportional durations (in \%) within each group: wild type $(n=12)$ and $P \operatorname{eg} 13^{-1-}(n=12)(* P<0.05, * * P<0.01$, and $* * * P<0.001)$. ( $(B)$ Selectivity plots. The selectivity index measures the strength of preference over the whole duration of the experiment, independent of the preference (18). Note that these plots include a higher resolution of time points. Error bars represent the variability of the selectivity index score within each group at each time point.

Changed Gene Regulation. Gene Ontology (GO) analysis on the set of genes that are affected in both sexes revealed an enrichment of loci involved in basic brain pathways and functions, including synapse formation (serotonergic, GABAergic, and glutamatergic), kinase activity, ion transport, and ion channel activity. Among the many genes that fall into these classes, we selected a set that showed particularly strong changes in the RNA-sequencing (RNA-seq) dataset, and that represents wellstudied genes in these pathways. For this set, we conducted qPCR experiments, including all mutant genotypes. The results are summarized in Fig. 4 and we discuss them in more detail in the following.

Serotonin-based signaling pathways play a crucial role in brain function and the regulation of numerous behaviors including sexual behavior and maternal care $(20,21)$. The expression of the brain-specific tryptophan hydroxylase (Tph2), the rate-limiting enzyme in the biosynthesis of serotonin, is significantly lowered in the Peg $13^{-/-}$and $\mathrm{Peg}_{1} 3^{+/-}$mice (Fig. 4). Combined changes in
Tph2 level and serotonin receptors have been shown to affect sexual behavior $(22,23)$, and we also observe significant changes in the serotonin receptors-especially Htrla (highly up-regulated) and Htrlb (down-regulated) (Fig. 4). The changes in serotonin-mediated signaling could also explain the observed changes in anxiety behavior for the Peg13 mutant mice, since serotonin is known to control these pathways.

Parenting behavior is mediated via GABAergic neurons in the medial amygdala (24). GABA is primarily synthesized from glutamate via the enzyme glutamate decarboxylase (GAD), encoded by Gad1 and Gad2. We find that Gad1 is significantly lowered in the Peg $13^{-/-}$and $P e g 13^{+/-}$mice of both sexes, but only in males of both genotypes for Gad2 (Fig. 4). Mice use olfactory cues to distinguish sex, social, or reproductive status (25) and this is mediated via glutamatergic synapses. We find that the expression of the glutaminase gene $(G l s)$ and eight different glutamate receptors (Gria1, Gria2, Gria3, Gria4, Grin1, Grin2c, Grik3, Grik5) with known functions at olfactory nerve 


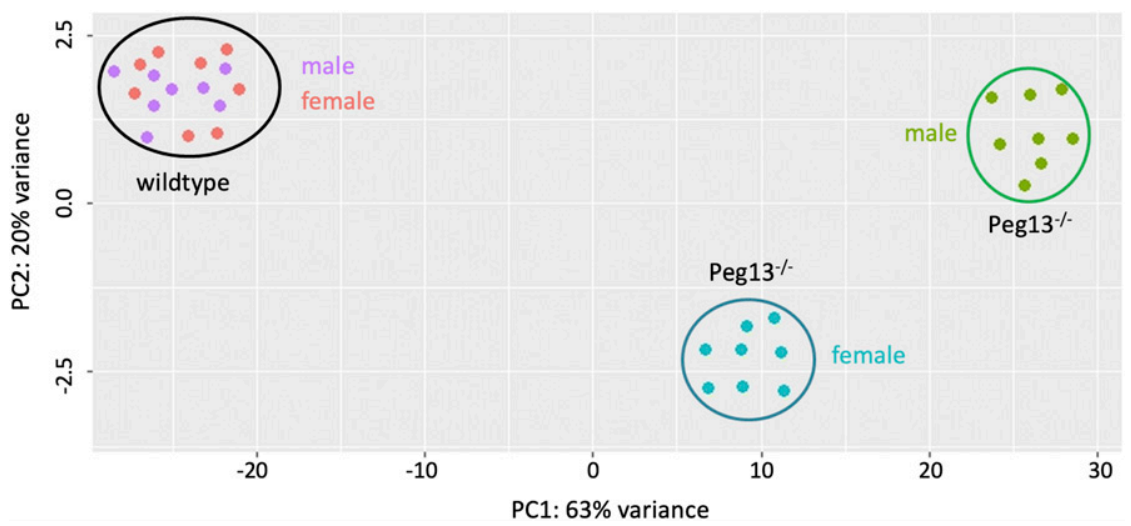

Fig. 3. PCA on the transcriptome samples of WT and Peg13 mutant mouse brains. WT individuals form a tight cluster, independent of the sex of the animals. Mutant mice form two distinct clusters-one for males, the other for females.

synapses is significantly lowered in the $P e g 13^{-/-}$and $P e g 13^{+/-}$ mice of both sexes (Fig. 4).

Approximately $60 \%$ of the expression of other known imprinted genes is significantly changed (Dataset $\mathrm{S} 1 K$ ). This includes several for which behavioral effects were inferred from previous knockout studies (listed in Fig. 4), implying that Peg13 is part of a regulatory network involving also other imprinted regions.

Intriguingly, the principal-component analysis (PCA) in Fig. 3 shows that while WT male and female samples group together, $P e g 13^{-/-}$male and female samples split into different groups. This suggests that their brain transcriptomes differ more from each other than those among WT mice. Indeed, we find no significant differentially expressed genes between male and female WT samples, while 629 genes show a significant difference in their expression between female and male Peg13 $13^{-1}$ mice (Dataset S1 $F$ and $G$ ). These include genes involved in estrogen signaling and other neuroreceptors (see also Fig. 4, male vs. female differences).

The estrogen signaling pathway plays an important role in neuronal development, in particular in sexually dimorphic regions, such as the anteroventral periventricular nucleus of the hypothalamus and the medial preoptic area (26). Estrogen mediates many of its effects through the activation of its receptors, including the $\mathrm{G}$ protein-coupled estrogen receptor Gper1. Expression of this receptor is significantly lowered in Peg13 mutant males compared with Peg13 mutant females (Fig. 4).

There are also a number of important neuroreceptors that are differentially expressed between the sexes in Peg13 mutant mice. These include the serotonin receptor Htr2a (lower in males only, compared with WT), the opioid receptor Oprm1, and the galanin receptor Galr2. Galanin neurons in the preoptic area were shown to have a specific effect on parental behavior (27). The DOPA decarboxylase $D d c$, required for synthesizing dopamine and serotonin, is also highly asymmetrically affected, being reduced only in males compared with WT (Fig. 4).

For all of the genes tested, we find congruent results for homozygous mutant individuals and individuals that have inherited the mutant allele from the father (Fig. 4). Conversely, all individuals that have inherited the mutant allele from the mother show WT-like expression. This serves as an additional confirmation that the effects are due to the disruption of Peg13, rather than an indirect effect from the flanking genes that are all expressed from the maternal chromosome.

\section{Discussion}

The adult brain is generally strongly shaped by the action of imprinted genes, including specific effects on sexual behavior, parental care, foraging, and exploration of novel environments, and several different areas of the brain mediate this (28). Peg13 is broadly expressed throughout the brain (12) and may therefore exert a basic function in the differentiation of neurons that mediate specific behavioral responses (29). The broad effects on the transcriptome may be partly due to its transregulatory effects on other imprinted loci, which may further influence the interaction network and neural circuits that set up the social brain (28). The functional consequences of the partial Peg13 disruption are nonetheless rather specific. We do not simply observe phenotypes that could be explained by a loss of basic signaling functions in the brain since, for the sex preference, we see a reversal. The animals still show sex preferences, but for the same sex instead of the opposite sex. Interestingly, we also observe nest-building behavior when same-sex mutant animals share a cage (SI Appendix, S4). When opposite sexes share a cage, they occasionally mate successfully and produce normal-sized litters. A large part of these litters is lost, apparently due to lack of maternal care, which is a phenotype that was also found for three other imprinted loci $(2,30)$. One of these is Peg3, and this is significantly down-regulated in Peg13 mutant mice (Fig. 4), that is, the corresponding Peg13 maternal care phenotype might be an indirect effect of this down-regulation.

Several lncRNAs are known to control imprinting clusters (1) and interactions may occur through specific genomic sites by forming triplexes with the DNA duplex, through which they recruit DNA- and histone-modifying enzymes to these sites (31, 32). However, there are a multitude of possible regulatory mechanisms of lncRNAs (33) and the molecular mechanisms of action of Peg13 therefore remain open at present, especially because of its fast evolution that does not allow identifying possible functional domains.

The stronger male-versus-female brain transcriptomic difference in Peg $13^{-/-}$mice is an unexpected phenotype. There have been controversial discussions about sex dimorphisms in the brain and whether these are genetically and/or environmentally determined. One can trace some sex differences in different brain regions, and this has led to the "mosaic" hypothesis with different degrees of "maleness" or "femaleness" within a single brain (34). However, given that our transcriptome analysis was across the whole brain, this appears to average out, explaining why we do not see significant differences between WT male and female whole-brain transcriptomes. The loss of Peg13 gene function surprisingly leads to a higher differentiation of the male-versus-female brain. This could be explained if there is early in development a split of the pathways that determine male-versus-female brain differentiation, whereby the Peg13mediated pathway would work toward making the brains of the two sexes more similar on average. 


\begin{tabular}{|c|c|c|c|c|c|}
\hline & & \multicolumn{2}{|c|}{ WT vs. mutant } & \multicolumn{2}{|l|}{$\begin{array}{l}\text { male vs. } \\
\text { female }\end{array}$} \\
\hline & & male & female & \multirow{3}{*}{\multicolumn{2}{|c|}{ 々 }} \\
\hline gene & & & & & \\
\hline symbol & gene name & है & 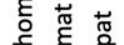 & & \\
\hline \multicolumn{6}{|c|}{ enzymes for neuroreceptor turnover } \\
\hline Tph2 & tryptophan hydroxylase 2 & & & & \\
\hline Gad1 & glutamate decarboxylase 1 & & & & \\
\hline Gad2 & glutamate decarboxylase 2 & & & & \\
\hline$D d c$ & dopa decarboxylase & & & & \\
\hline Gls & glutaminase & & & & \\
\hline \multicolumn{6}{|c|}{ neuroreceptors } \\
\hline Htr1a & serotonin receptor $1 \mathrm{~A}$ & & & & \\
\hline$H \operatorname{tr} 1 b$ & serotonin receptor $1 \mathrm{~B}$ & & & & \\
\hline Htr2a & serotonin receptor $2 \mathrm{~A}$ & & & & \\
\hline Gper1 & G-protein-coupled estrogen receptor 1 & & & & \\
\hline Galr2 & Galanin receptor 2 & & & & \\
\hline Oprm1 & Opioid receptor, mu 1 & & & & \\
\hline Gria1 & glutamate receptor, AMPA1 (alpha 1) & & & & \\
\hline Gria2 & glutamate receptor, AMPA2 (alpha 2) & & & & \\
\hline Gria3 & glutamate receptor, AMPA3 (alpha 3) & & & & \\
\hline Gria4 & glutamate receptor, AMPA4 (alpha 4) & & & & \\
\hline Grin1 & glutamate receptor, NMDA1 (zeta 1) & & & & \\
\hline Grin2c & glutamate receptor, NMDA2C (epsilon 3) & & & & \\
\hline Grik3 & glutamate receptor, kainate 3 & & & & \\
\hline Grik5 & glutamate receptor, kainate 5 (gamma 2) & & & & \\
\hline \multicolumn{6}{|c|}{ imprinted loci with known behavioral phenotypes } \\
\hline Peg3 & paternally expressed 3 & & & & \\
\hline Ube3a & ubiquitin protein ligase E3A & & & & \\
\hline Snrpn & small nuclear ribonucleoprotein $\mathrm{N}$ & & & & \\
\hline Rasgrf1 & RAS guanine nucleotide-releasing factor 1 & & & & \\
\hline Grb10 & growth factor receptor bound protein 10 & & & & \\
\hline Zrsr1 & zinc finger ( $\mathrm{CCCH}$ type) & & & & \\
\hline Commd1 & COMM domain containing 1 & & & & \\
\hline Dlk1 & delta like non-canonical Notch ligand 1 & & & & \\
\hline Meg3 & maternally expressed 3 & & & & \\
\hline
\end{tabular}

Fig. 4. Genes with significant transcriptome changes between mutant and WT mice. Results from qPCR experiments of a subset of genes that were found to be differentially expressed in the RNA-seq analysis. Genes that show a significant $(P<<0.001)$ expression change in the different genotypes are marked with colored boxes: red for lowered expression, and blue for enhanced expression. White boxes represent no significant change. Genotype designations: hom,

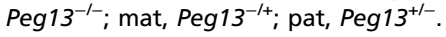

The strong paternal imprint of Peg13 is conserved between mice and humans, although neither the sequence nor most of the imprinting status of the other genes in the cluster is conserved. Hence, one can expect a strong evolutionary pressure to retain the paternal imprinting status of Peg13 and its modulation of male-female brain differentiation may play a role in this. The evolution of imprinting was originally modeled within the framework of kinship theory (35), but the predictions of this model covered mostly the developmental conflict aspects in fetal and placental tissues. A new model based on bet hedging and reproductive variance consideration suggests that paternally expressed imprinted genes would promote risk-averse behaviors that reduce reproductive variance, especially in males (36). Hence, the modulatory effect of Peg13 making the brains more similar between the sexes could be interpreted in the direction of making the male brain more female-like.

Even this theory cannot explain why we see a change in sexual preference in Peg1 $3^{-/-}$mice. The genetic basis for sexual preference has been much discussed (37), with a recent comprehensive genome-wide association study in humans being unable to reveal major loci associated with same-sex sexual behavior (38). There is also no association peak in the region of chromosome 8 that encodes the Peg13 locus in humans. However, given the fast evolution of the Peg13 RNA sequence, it may well be that each naturally segregating single-nucleotide polymorphism variant only has a minor effect on the phenotype. Moreover, the statistics used in the above study did not correct for imprinted loci, that is, a possible association would likely have been missed. Even if a deeper statistical analysis would reveal Peg13 to be involved in human sexual preference, at least in mice, its functional role is much broader. With its trans effects on many other imprinted loci in the brain, it is likely a key regulator for the development of the neural circuits of social behavior (29).

In view of its broad regulatory roles, it is surprising that Peg13 evolves so fast, not much slower than neutral intergenic DNA. It could thus indeed contribute to fast behavioral divergence patterns of natural mouse populations, as we have speculated previously (10). Given that assortative mating patterns can significantly speed up adaptive speciation processes $(39,40)$, 
the fast evolution of Peg13 could also play an important role in mammalian speciation patterns.

\section{Materials and Methods}

Animal Experimentation. The behavioral studies were approved by the supervising authority (Ministerium für Energiewende, Landwirtschaftliche Räume und Umwelt, Kiel, Germany) under registration nos. V244-71173/ 2015, V244-4415/2017, and V244-47238/17. Animals were kept according to Federation of European Laboratory Animal Science Association guidelines, with the permit from Veterinäramt Kreis Plön: 1401-144/PLÖ-004697. The respective animal welfare officer at the University of Kiel was informed about the killing of the animals for this study. All mice were bred in type II cages (Bioscape) and were weaned at the age of $3 \mathrm{wk}$. At weaning, the sexes were separated. Males were housed together with brothers or in individual cages. Females were housed in sister groups to a maximum of five mice per cage. Enrichment, including wood wool, paper, egg cartons, and a spinning wheel (Plexx), was provided in each cage. Mice were fed standard diet 1324 (Altromin) and provided water ad libitum. Housing was at 20 to $24{ }^{\circ} \mathrm{C}$, with 50 to $65 \%$ humidity, and on a 12-h:12-h light-dark schedule with lights on at 7 AM. All behavioral experiments were done by experimenters blinded to the genotype.

Knockout. The knockout strategy was discussed in conjunction with the company genOway (https://www.genoway.com/), which conducted the knockout and delivered the mice. In short, a neomycin selection cassette was used to replace the $3^{\prime}$ part of the Peg13 transcript and LoxP sites flanking the whole gene were added to allow a full deletion on top of the partial deletion. The construct was inserted via homologous recombination into mouse embryonic stem cells. After homologous recombination, the neomycin selection cassette was removed through Flp-mediated recombination to create Peg13 ${ }^{\text {tm } 1 M e v b}$. Additional Cre-mediated recombination (17) was used to create Peg $13^{\text {tmi.1Mevb }}$. Further details are provided in SI Appendix, S2. The initial analysis of the knockout mice was done in the context of the thesis of M.K. (41)

Behavioral Tests. The behavioral tests described below were filmed using a TSE camera (TSE Systems) from the top of the setups, for 5 min each. Setups were cleaned with $30 \%$ ethanol after each test. The videos were transferred to the VideoMot2 System (TSE Systems) which allows automated tracking of the mice. Individuals were detected by the software at three points (head/center/tail base tracking) and then the software automatically generated the numerical data of the time that each mouse spent at zones of interest.

Elevated plus maze. Mice were placed in the center of an elevated plus maze (arms are $50 \times 50 \mathrm{~cm}$ with a $10-\mathrm{cm}$ neutral area in the center where the arm cross). Two of the arms were made of clear Plexiglas and two were made of gray polyvinyl chloride (PVC). The floor was made of white PVC. The time spent in the dark arms was measured as anxiety index.

Open field. Mice were placed in a $60 \times 60-\mathrm{cm}$ open box and allowed to explore the area for $5 \mathrm{~min}$. The distance traveled and time spent within $10 \mathrm{~cm}$ of the wall vs. in the central area was used as activity and anxiety index.

Dark/light box. The focal mouse was placed in a test box $(20 \times 30 \times 10 \mathrm{~cm})$ containing a small dark shelter with two exits. The time until the mouse tail became visible was recorded.

Novel object. The test was performed in the same test apparatus as the open field test, but this time a LEGO toy was placed in the center as a novel object. The length of time of the novel object being explored was measured. All data are provided in Dataset S2A.

Pup Retrieval Test. Testing was performed in the home cage of female mice (eight per genotype). The females were mated with wild-type males, which were removed before conception. Three days after giving birth, the mother was removed from the cage shortly before the behavioral test and offspring were divided into two groups and placed in different corners opposite the nest. Pup retrieval was defined as picking a pup up in the mouth and transporting it to the nest. Latencies to retrieve each pup to the nest were recorded during the first $15 \mathrm{~min}$. The latencies to retrieve the first two pups to the nest were used for statistical purposes and knockout females that did not retrieve the pups were assigned a latency of $900 \mathrm{~s}$ (15 min). All data are provided in Dataset $\mathrm{S} 2 B$.

Partner Preference Test. The partner preference assay was done as described (18) using individuals with no prior contact with the other sex after weaning. The experimental setup consisted of five standard Makrolon cages
(Techniplast): one central cage $(40.5 \times 28.0 \times 20.0 \mathrm{~cm})$ and four type III satellite cages, which were connected via Plexiglas tubes to each one of the four sides of the central cage. Each Plexiglas connection was equipped with two RFID ring antennae (TSE Industries), one close to the central cage and one close to the respective satellite cage. Each mouse was equipped with an RFID tag (Iso FDX-B; Planet ID), which was read every time the mouse passed one of the antennae. The satellite cages were divided into two parts by a metal grid. This setup allowed the focal mouse to interact (i.e., via smell and vocalization), but copulation was prevented. Each group of satellite mice, consisting of four mice of both sexes and different genotypes (wild type and knockout), changed over the experiments for each focal mouse and positions of cage occupation were randomized. Water and food as well as bedding were provided in all cages.

Each run of the experiment began by placing the focal and satellite mice in their respective cages and starting the computer program monitoring the RFID antennae. Each experiment lasted for $3 \mathrm{~h}$. For each focal individual a text file was generated with time stamps for every antenna read and the identification number of the respective antenna. Using a script in $\mathrm{R}$, we calculated the duration of time spent in the four satellite cages. To analyze the selectivity (i.e., the strength of preference that focal mice show for some satellite mice over others, independent of the direction of preference), we calculated a selectivity index $(\mathrm{SI})$ with the formula $\mathrm{SI}=\mathrm{SD} / \mathrm{SD}(\max )$, where SD is the SD of the four proportional durations (in \%) that the respective focal mouse spent in each of the four satellite cages, and $S D(\max )$ is the maximal SD theoretically possible, which is in our case (four possibilities) 50 . The resulting SI values range from 0 ( $25 \%$ of the time in each of the four satellite cages $=$ no selectivity) to $1(100 \%$ of the time in one of the four satellite cages, no time in the three others = highest selectivity). SI was calculated in 10-min intervals for each focal mouse to get measures for the change of selectivity over time, and over the whole period to get a measure of overall selectivity for each individual. Note that ref. 18 had found that selectivity in wild mice is only established after $1 \mathrm{~d}$ in this setup. We had therefore done initial tests with longer runs of the experiment, but found that for the laboratory strain that was used here, a 3-h interval is sufficient to display the main patterns of selectivity. All data are provided in Dataset S2C.

RNA Extraction. RNA extraction (from whole brain) was done using TRIzol reagent. One milliliter of TRIzol per $40 \mathrm{mg}$ tissue was added to each sample. The samples were lysed on a Tissuelyser II (QIAGEN) at $30 \mathrm{~Hz}$ for $5 \mathrm{~min}$. Homogenized samples were incubated at room temperature for $5 \mathrm{~min}$. Two hundred microliters of chloroform per $1 \mathrm{~mL}$ TRIzol was added to each sample and shaken vigorously by hand for $15 \mathrm{~s}$, followed by a 3-min incubation at room temperature and spinning at $12,000 \times g$ for $15 \mathrm{~min}$ at $4{ }^{\circ} \mathrm{C}$. The aqueous phase was transferred to a new tube and 0.5 volume of isopropanol was added, incubated at room temperature for $10 \mathrm{~min}$, and spun at 12,000 $\times$ $g$ at $4{ }^{\circ} \mathrm{C}$ (the RNA forms a gel-like precipitate). The supernatant was removed and the pellet was washed with $75 \%$ EtOH (made with RNase-free water). Samples were mixed by hand several times and then spun at 7,500 $\times$ $g$ for $5 \mathrm{~min}$ at $4{ }^{\circ} \mathrm{C}$. The supernatant was removed and the pellet was dried briefly at room temperature, dissolved in $200 \mu \mathrm{L}$ RNase-free water, and stored at $-20^{\circ} \mathrm{C}$ overnight. An equal volume of $\mathrm{LiCl}(5 \mathrm{M})$ was added to the crude RNA extract, mixed by hand, and incubated for $1 \mathrm{~h}$ at $-20^{\circ} \mathrm{C}$. Samples were spun at $16,000 \times g$ for $30 \mathrm{~min}$. The supernatant was removed, and samples were washed twice with $70 \% \mathrm{EtOH}$ and spun at $10,000 \times g$ at $4{ }^{\circ} \mathrm{C}$. The pellet was dried at room temperature, dissolved in RNase-free water, and kept at $-70^{\circ} \mathrm{C}$.

RNA-Seq Analysis. RNA sequencing was performed on 16 Peg $13^{-/-}$and 16 WT mice from the same breeding stock ( 8 virgin females and 8 males in each group) at the age of $16 \mathrm{wk}$. The animals were derived from the offspring of 12 heterozygous parental pairs, with a maximum of three animals from the same litter. The sequencing of the samples was performed using a poly(A) tail purification step, followed by complementary DNA (cDNA) synthesis and library preparation by using the TruSeq stranded RNA HT Kit from Illumina. Libraries passing quality control were subjected to sequencing on an Illumina HiSeq 2000 sequencer. Each transcriptome sample was sequenced in approximately one-third of a HiSeq 2000 lane. Raw sequence reads were quality trimmed using Trimmomatic (42). The quality trimming was performed base-wise, removing bases below a quality score of 20 (Q20), and keeping reads whose average quality was at least Q60. Reads were mapped to the mouse mm10 reference genome (Genome Sequencing Consortium 2002) by using HISAT2 (43). Between 19.8 and 23.7 million mapped reads were obtained per sample. HTSeq was used for counting reads overlapping into a specific feature (gene) (44). Differential expression analysis and PCA 
were performed with the DESeq2 package (19) in an R environment. GO and Kyoto Encyclopedia of Genes and Genomes (KEGG) pathway enrichment analyses were performed using DAVID online tools (version 6.8), with the classification stringency set to a "medium" $P$ value of $<0.05$. The RNA-seq data were deposited in the European Nucleotide Archive (ENA) under accession no. PRJEB39594.

qPCR. RNA extraction was done by using TRIzol reagent as described above from 16 WT and 16 each Peg $13^{-/-}, 16$ Peg $13^{+/-}$, and 16 Peg $13^{-/+}$(8 females and 8 males in each group). One microgram of high-quality total RNA from each brain sample was used for CDNA synthesis. CDNA was synthesized using the First-Strand CDNA Synthesis Kit according to the instructions of the supplier (Thermo Fisher Scientific). Quantitative digital PCR (45) was performed for a subset of genes found to be significantly differentially expressed in the RNA-seq analysis. The primers (SI Appendix, S5) were designed with a GC content $<50 \%$ and with very low potential for primer dimer structure. The QX100 Droplet Digital PCR System (Bio-Rad) was used according to the manufacturer's instructions. Briefly, fluorescent PCRs for each sample were prepared in a $24-\mu \mathrm{L}$ volume containing $12 \mu \mathrm{L} 2 \times$ EvaGreen supermixes, $200 \mathrm{nM}$ each forward and reverse primer, cDNA, and water. Droplets were generated using a droplet generator (DG) with an eightchannel DG8 cartridge and cartridge holder with $70 \mu \mathrm{L}$ DG oil per well, $20 \mu \mathrm{L}$ fluorescent PCR mixture, and a DG8 gasket. The prepared droplets were transferred to corresponding wells of a 96-well PCR plate (Eppendorf). The PCR plate was subsequently heat sealed with pierceable foil using a PX1 PCR plate sealer (Bio-Rad) and then amplified in a LifeEco thermal cycler (Bioer). The thermocycling protocol was as follows: initial denaturation at $95^{\circ} \mathrm{C}$ for $5 \mathrm{~min}$, then 40 cycles of denaturation at $95^{\circ} \mathrm{C}$ for $30 \mathrm{~s}$, annealing at $60{ }^{\circ} \mathrm{C}$ for $45 \mathrm{~s}$, and, finally, incubation first at $4{ }^{\circ} \mathrm{C}$ for $5 \mathrm{~min}$ and then at $90^{\circ} \mathrm{C}$ for $5 \mathrm{~min}$. After cycling, the 96-well plate was fixed in a plate holder and placed in the droplet reader. Droplets of each sample were analyzed sequentially and fluorescent signals of each droplet were measured individually by a detector. The data analysis was performed with QuantaSoft droplet reader software (Bio-Rad). For each gene, the software calculated the absolute concentration of CDNA as copies per microliter. For the correction of the input CDNA, the calculated value for each gene was normalized by the expression of $\beta$-catenin as reference gene in each sample.

Brain Analysis by Micro Cr. Mutant and wild-type mice were killed at the age of $18 \mathrm{wk}$. The brain was dissected from the skull and subsequently fixed in

1. D. P. Barlow, M. S. Bartolomei, Genomic imprinting in mammals. Cold Spring Harb. Perspect. Biol. 6, a018382 (2014).

2. L. Lefebvre et al., Abnormal maternal behaviour and growth retardation associated with loss of the imprinted gene Mest. Nat. Genet. 20, 163-169 (1998).

3. L. Li et al., Regulation of maternal behavior and offspring growth by paternally ex pressed Peg3. Science 284, 330-333 (1999).

4. A. S. Garfield et al., Distinct physiological and behavioural functions for parental alleles of imprinted Grb10. Nature 469, 534-538 (2011)

5. C. L. Dent et al., Impulsive choice in mice lacking paternal expression of Grb10 suggests intragenomic conflict in behavior. Genetics 209, 233-239 (2018).

6. A. Plagge et al., Imprinted Nesp55 influences behavioral reactivity to novel environments. Mol. Cell. Biol. 25, 3019-3026 (2005).

7. C. L. Dent et al., Impulsive choices in mice lacking imprinted Nesp55. Genes Brain Behav. 15, 693-701 (2016)

8. C. N. Stacher Hörndli et al., Complex economic behavior patterns are constructed from finite, genetically controlled modules of behavior. Cell Rep. 28, 1814-1829.e6 (2019).

9. I. Montero, M. Teschke, D. Tautz, Paternal imprinting of mating preferences between natural populations of house mice (Mus musculus domesticus). Mol. Ecol. 22, 2549-2562 (2013).

10. A. Lorenc, M. Linnenbrink, I. Montero, M. B. Schilhabel, D. Tautz, Genetic differentiation of hypothalamus parentally biased transcripts in populations of the house mouse implicate the Prader-Willi syndrome imprinted region as a possible source of behavioral divergence. Mol. Biol. Evol. 31, 3240-3249 (2014).

11. R. J. Smith, W. Dean, G. Konfortova, G. Kelsey, Identification of novel imprinted genes in a genome-wide screen for maternal methylation. Genome Res. 13, 558-569 (2003).

12. W. Davies, R. J. Smith, G. Kelsey, L. S. Wilkinson, Expression patterns of the novel imprinted genes Nap115 and Peg13 and their non-imprinted host genes in the adult mouse brain. Gene Expr. Patterns 4, 741-747 (2004).

13. F. Court et al., The PEG13-DMR and brain-specific enhancers dictate imprinted ex pression within the $8 \mathrm{q} 24$ intellectual disability risk locus. Epigenetics Chromatin 7, 5 (2014).

14. P. Singh et al., Chromosome-wide analysis of parental allele-specific chromatin and DNA methylation. Mol. Cell. Biol. 31, 1757-1770 (2011). freshly prepared $4 \%$ paraformaldehyde. The sample was dehydrated through graded alcohols $(12 \mathrm{~h})$ and stained in $1 \%$ iodine solution in $90 \%$ methanol (46). After $24 \mathrm{~h}$ in the solution, the sample was rinsed in an ethanol rehydration series to end up in sterile distilled water. For increased stability in the micro-CT instrument, the brain was embedded in $2 \%$ agarose gel and placed in a plastic tube. Micro CT was performed with a Bruker SkyScan 1276 (RJL Micro \& Analytic). The micro-CT scan was carried out at 90 $\mathrm{kV}$ acceleration voltage and $40 \mu \mathrm{A}$ tube current. Exposure time was $2,120 \mathrm{~ms}$ and five images were averaged for reducing the noise. Reconstruction was performed by NRecon software (RJL Micro \& Analytic). Reconstructed data were imported into Dataviewer (RJL Micro \& Analytic) for visualization and further analysis. In this software, the three-dimensional data were aligned so that orthogonal slices matched the horizontal, coronal, and sagittal planes, resulting in series of 1,100 coronal, 770 sagittal, and 480 horizontal sections of the brain. These data were compared with an anatomical atlas (Allen Mouse Brain Atlas, Allen Institute for Brain Science@2004, mouse. brain-map.org/static/atlas) for further analysis. https://zenodo.org/record/ 4450416\#.YA_71hJ7laR.

Data Availability. The RNA-seq data reported in this article have been deposited in the European Nucleotide Archive (ENA) (accession no. PRJEB39594). The CT data are deposited at https://zenodo.org/record/ 4450416\#.YA 71hJ7laR (doi:10.5281/zenodo.4450416).

ACKNOWLEDGMENTS. We thank Guy Reeves and Luisa Pallares for comments on the manuscript; Chen Xie for his valuable advice with tran scriptome analysis; Ana Teles, Peter Refki, Michael Schwarz, and Anja Baade for their help in tissue sectioning and staining; Yoland Savriama and Elke Blohm-Sievers for help with the CT experiments; Sven Künzel for sequencing Rebecca Krebs-Wheaton and Anja Guenther for their advice on the behavioral experiments; Barbara Kleinhenz and Fabian Boesel for their great laboratory-related support; and the entire mouse team at the Max Planck Institute for Evolutionary Biology, in particular Christine Pfeifle, Anastasia Volk, Anika Jonas, Heike Harre, Susanne Holz, and Camilo Medina for taking care of the mice and also running the behavioral tests. Portions of this paper were developed from the dissertation of M.K., University of Kiel, Germany. The project was funded by institutional resources of the Max Planck Society (D.T.). The funder had no influence on the experimental design or interpretation.

15. S. Suzuki, G. Shaw, T. Kaneko-Ishino, F. Ishino, M. B. Renfree, The evolution of mammalian genomic imprinting was accompanied by the acquisition of novel $\mathrm{CpG}$ islands. Genome Biol. Evol. 3, 1276-1283 (2011).

16. W. Xie et al., Base-resolution analyses of sequence and parent-of-origin dependent DNA methylation in the mouse genome. Cell 148, 816-831 (2012).

17. M. Lakso et al., Efficient in vivo manipulation of mouse genomic sequences at the zygote stage. Proc. Natl. Acad. Sci. U.S.A. 93, 5860-5865 (1996)

18. M. Linnenbrink, S. von Merten, No speed dating please! Patterns of social preference in male and female house mice. Front. Zool. 14, 38 (2017).

19. M. I. Love, W. Huber, S. Anders, Moderated estimation of fold change and dispersion for RNA-seq data with DESeq2. Genome Biol. 15, 550 (2014).

20. B. Olivier, Serotonin: A never-ending story. Eur. J. Pharmacol. 753, 2-18 (2015).

21. M. Angoa-Pérez, D. M. Kuhn, Neuroanatomical dichotomy of sexual behaviors in rodents: A special emphasis on brain serotonin. Behav. Pharmacol. 26, 595-606 (2015)

22. E. M. S. Snoeren, J. G. Veening, B. Olivier, R. S. Oosting, Serotonin 1 A receptors and sexual behavior in female rats: A review. Pharmacol. Biochem. Behav. 121, 43-52 (2014).

23. B. Olivier et al., Differences in sexual behaviour in male and female rodents: Role of serotonin. Curr. Top. Behav. Neurosci. 8, 15-36 (2011).

24. P. B. Chen et al., Sexually dimorphic control of parenting behavior by the medial amygdala. Cell 176, 1206-1221.e18 (2019).

25. K. R. Kelliher, S. R. Wersinger, Olfactory regulation of the sexual behavior and re productive physiology of the laboratory mouse: Effects and neural mechanisms. ILAR J. 50, 28-42 (2009).

26. R. B. Simerly, Wired for reproduction: Organization and development of sexually dimorphic circuits in the mammalian forebrain. Annu. Rev. Neurosci. 25, 507-536 (2002).

27. Z. Wu, A. E. Autry, J. F. Bergan, M. Watabe-Uchida, C. G. Dulac, Galanin neurons in the medial preoptic area govern parental behaviour. Nature 509, 325-330 (2014)

28. J. D. Perez, N. D. Rubinstein, C. Dulac, New perspectives on genomic imprinting, an essential and multifaceted mode of epigenetic control in the developing and adult brain. Annu. Rev. Neurosci. 39, 347-384 (2016).

29. P. Chen, W. Hong, Neural circuit mechanisms of social behavior. Neuron 98, 16-30 (2018) 
30. H. D. J. Creeth, G. I. McNamara, A. R. Isles, R. M. John, Imprinted genes influencing the quality of maternal care. Front. Neuroendocrinol. 53, 100732 (2019).

31. J. T. Lee, Lessons from X-chromosome inactivation: Long ncRNA as guides and tethers to the epigenome. Genes Dev. 23, 1831-1842 (2009).

32. M. C. Tsai et al., Long noncoding RNA as modular scaffold of histone modification complexes. Science 329, 689-693 (2010).

33. J. D. Ransohoff, Y. Wei, P. A. Khavari, The functions and unique features of long intergenic non-coding RNA. Nat. Rev. Mol. Cell Biol. 19, 143-157 (2018).

34. D. Joel, A. Garcia-Falgueras, D. Swaab, The complex relationships between sex and the brain. Neuroscientist 26, 156-169 (2020)

35. J. F. Wilkins, D. Haig, What good is genomic imprinting: The function of parentspecific gene expression. Nat. Rev. Genet. 4, 359-368 (2003).

36. J. F. Wilkins, T. Bhattacharya, Intragenomic conflict over bet-hedging. Philos. Trans. $R$. Soc. Lond. B Biol. Sci. 374, 20180142 (2019)

37. M. C. Mills, How do genes affect same-sex behavior? Science 365, 869-870 (2019).

38. A. Ganna et al., Large-scale GWAS reveals insights into the genetic architecture of same-sex sexual behavior. Science 365, eaat7693 (2019)
39. U. Dieckmann, M. Doebeli, On the origin of species by sympatric speciation. Nature 400, 354-357 (1999).

40. M. Doebeli, U. Dieckmann, J. A. Metz, D. Tautz, What we have also learned: Adaptive speciation is theoretically plausible. Evolution 59, 691-695; discussion, 696-699 (2005).

41. M. Keshavarz, "Analysis of candidate genes for behavioral differences in mice," PhD thesis, Christian Albrechts Universität zu Kiel, Kiel, Germany (2018).

42. A. M. Bolger, M. Lohse, B. Usadel, Trimmomatic: A flexible trimmer for Illumina sequence data. Bioinformatics 30, 2114-2120 (2014).

43. D. Kim, B. Langmead, S. L. Salzberg, HISAT: A fast spliced aligner with low memory requirements. Nat. Methods 12, 357-360 (2015).

44. S. Anders, P. T. Pyl, W. Huber, HTSeq-A Python framework to work with highthroughput sequencing data. Bioinformatics 31, 166-169 (2015).

45. P. L. Quan, M. Sauzade, E. Brouzes, dPCR: A technology review. Sensors (Basel) 18, 1271 (2018).

46. T. Zikmund et al., High-contrast differentiation resolution 3D imaging of rodent brain by X-ray computed microtomography. J. Instrum. 13, C02039-C02039 (2018). 